\title{
Increased COMT expression in pancreatic cancer and correlation with clinicopathologic parameters
}

\author{
WU WenMing ${ }^{1 \dagger}$, ZHANG Jie $^{1 \dagger}$, ZHOU Li $^{1}$, YOU Lei ${ }^{1}$, ZHAO YuPei ${ }^{1 *} \& \mathrm{LI} \mathrm{Ji}^{2}$ \\ ${ }^{1}$ Department of General Surgery, Peking Union Medical College Hospital, Chinese Academy of Medical Sciences and \\ Peking Union Medical College, Beijing 100730, China; \\ ${ }^{2}$ Department of Pathology, Peking Union Medical College Hospital, Chinese Academy of Medical Sciences and \\ Peking Union Medical College, Beijing 100730, China
}

Received June 16, 2012; accepted August 22, 2012

\begin{abstract}
Catechol-O-methyl transferase (COMT) is an enzyme involved in estrogen metabolism. Proteomic and immunoproteomic screens suggested COMT might be an immunogenic membrane antigen in human pancreatic cancer. The aim of this study was to investigate the dynamic expression of COMT in pancreatic ductal adenocarcinoma (PDAC) and noncancerous pancreatic tissue, and to determine the relationship between COMT expression and clinicopathologic parameters. COMT expression was analyzed by reverse transcription-polymerase chain reaction (RT-PCR) and western blot in five pancreatic cell lines and five pairs of PDAC and noncancerous pancreatic tissue. Immunohistochemistry was used to evaluate COMT expression in tissue microarrays and 20 cases of paraffin-embedded clinical specimens. The results indicated that COMT expression was detected in AsPC-1, BxPC-3, MIA PaCa-2, Capan-1 and SW1990 pancreatic cell lines, and in five pairs of PDAC and noncancerous pancreatic tissue, at the mRNA and protein levels. Immunohistochemistry analysis revealed that COMT expression was significantly higher in PDAC than in nonmalignant pancreatic tissue. High expression of COMT significantly correlated to early T stages. Therefore, we conclude that COMT might serve as a potential biomarker for applied clinical pathology in PDAC.
\end{abstract}

COMT, pancreatic cancer, immunohistochemistry, biomarker

Citation: Wu W M, Zhang J, Zhou L, et al. Increased COMT expression in pancreatic cancer and correlation with clinicopathologic parameters. Sci China Life Sci, 2012, 55: 747-752, doi: 10.1007/s11427-012-4375-y

Pancreatic cancer is the fourth leading cause of cancer-related mortality worldwide, and the death rate in females has increased in the last decade [1]. Radical surgical resection is one of the most important treatments to cure or prolong the survival of pancreatic cancer patients. However, only $10 \%-20 \%$ of all patients are suitable for potentially curative surgery $[2,3]$. Therefore, it is necessary to find tumor biomarkers for early diagnosis or tumor stage prediction. Currently the best marker available is carbohydrate antigen 19-9, which has a sensitivity of approximately $80 \%$ and thus is not adequate for screening purposes, particularly for the

$†$ Contributed equally to this work

*Corresponding author (email: yupeizhao_css@yahoo.com.cn) diagnosis of localized, resectable pancreatic cancers $[4,5]$.

In our previous studies [6,7], we screened membrane proteins from pancreatic cancer cell lines using two-dimensional electrophoresis, and performed immunoblotting with these proteins and serum IgG purified from clinically collected sera of pancreatic cancer patients. Proteomic and immunoproteomic results suggested that catechol-O-methyl transferase (COMT) might be a candidate immunogenic membrane antigen in human pancreatic cancer. Based on these findings, we became interested in the expression and function of COMT in pancreatic cancer.

The COMT gene is located at 22q11, and encodes two different mRNAs: a long membrane-bound COMT (MBCOMT) and a shorter soluble COMT (S-COMT) [8]. Pre- 
vious studies demonstrated that COMT is overexpressed in colorectal cancer [9], and have focused on the effects of different gene variants of COMT on the risk of hormone-dependent diseases, such as breast cancer [10-12], endometrial cancer [13], and gastric cancer [14]. However, the expression and function of COMT in pancreatic cancer has rarely been reported. Therefore, we aimed to investigate the expression of COMT in pancreatic cancer cell lines, human pancreatic ductal adenocarcinoma (PDAC) and nonmalignant pancreatic tissue, and to address the correlation between COMT expression and the clinicopathologic parameters.

\section{Materials and methods}

\subsection{Cell culture}

Human pancreatic cancer cell lines AsPC-1, BxPC-3, MIA PaCa-2, Capan-1 and SW1990 (all kind gifts from Professor Helmut Freiss, Technical University Munich, Germany) were maintained in $5 \% \quad \mathrm{CO}_{2}$ atmosphere at $37^{\circ} \mathrm{C}$ in Dulbecco's modified Eagle's medium or RPMI 1640 medium (Hyclone, Thermo Fisher Scientific Inc., Waltham, MA, USA), supplemented with $10 \%$ fetal bovine serum (Hyclone).

\subsection{Clinical samples and tissue microarrays}

Five paired cases of PDAC and adjacent healthy pancreatic tissue were collected from the Department of General Surgery of Peking Union Medical College Hospital (PUMCH). In addition, 20 paraffin-embedded tissue blocks of benign pancreatic-diseased tissue containing normal pancreatic tissue were obtained from the Pathology Department of PUMCH. Tissue microarrays with 80 paired sections of PDAC and adjacent para-carcinoma tissue were purchased from Shanghai Outdo Biotech Co. Ltd. (catalogue No. OD-CT-DgPan01-007). All of the human specimens in the study were approved by the ethics committee of PUMCH.

\subsection{Reverse transcription-polymerase chain reaction (RT-PCR)}

Total RNA was extracted using Trizol reagent (Invitrogen, Carlsbad, CA, USA) and cDNA was synthesized using an RT-PCR kit (Invitrogen) according to the manufacturer's instructions. The COMT primers used were as follows: 5'ATTGACACCTACTGCGAGCA-3' and 5'-CCACATTCCTCCAAGAGAAGC-3'. The internal control GAPDH primers were 5'-GGCTGCCATTTGCACTGGCAA-3' and 5'TGCCDTGAGTGGAGTCATACTG-3'. The PCR conditions were as follows: pre-denaturing at $94^{\circ} \mathrm{C}$ for $2 \mathrm{~min}, 30$ cycles of denaturing at $94^{\circ} \mathrm{C}$ for $30 \mathrm{~s}$, annealing at $60^{\circ} \mathrm{C}$ for $30 \mathrm{~s}$, and polymerization at $72^{\circ} \mathrm{C}$ for $30 \mathrm{~s}$, then extension for
5 min. The target bands were densitometrically analyzed using the UVISoft UVIBand Application V97.04 and FluorChem image analyzing software (Alpha Innotech, San Leandro, CA, USA), and the results were calculated as the ratio of the gray value relative to GAPDH.

\subsection{Western blotting}

Total protein was purified from pancreatic cancer cell lines and tissues, separated on 12\% SDS-acrylamide gels, and electrotransferred to polyvinylidene fluoride membranes (Millipore, Billerica, MA, USA). After blocking, the membranes were incubated overnight at $4^{\circ} \mathrm{C}$ with primary antibodies against COMT (1:500 dilution; Santa Cruz Biotechnology, Santa Cruz, CA, USA) and $\beta$-actin $(1: 2000$ dilution; Santa Cruz), followed by incubation with horseradish peroxidase (HRP)-conjugated anti-rabbit and anti-mouse secondary antibodies (1:2000 dilution; ZhongShan GoldenBridge Biotechnology, China) for 1 hour at room temperature. The blots were visualized with Immobilon ${ }^{\mathrm{TM}}$ Western (Millipore) and densitometrically analyzed using UVISoft UVIBand Application V97.04 and FluorChem (Alpha Innotech). The results were calculated as the ratio of the gray value relative to $\beta$-actin.

\subsection{Immunohistochemistry}

Tissue microarrays and 20 paraffin-embedded sections containing normal pancreas specimens were dewaxed and rehydrated in xylene and graded alcohol, followed by citrate buffer ( $\mathrm{pH}$ 6.0) retrieval using the high pressure method. After blocking peroxidase, the sections were incubated with anti-COMT primary antibody (1:800 dilution for tissue microarrays and $1: 200$ for slides) at $37^{\circ} \mathrm{C}$ for $2 \mathrm{~h}$. Next, the sections were treated with an HRP polymer kit, Polink-1 HRP Broad Bulk Kit (Golden Bridge International, Inc., Los Angeles, CA, USA) for signal amplification. Diaminobenzidine was used as the chromogen and the sections were counterstained with hematoxylin.

\subsection{Determination of COMT score}

According to published methods [15], the staining intensity of COMT was classified as absent: 0 ; weak: 1; medium: 2; and strong: 3. The percentage of positive cells was categorized as $0(<5 \%), 1(5 \%-25 \%), 2(>25 \%-50 \%), 3(>50 \%-$ $75 \%)$, and $4(>75 \%)$. By multiplying the value of the staining intensity by the categorized value of positive cells, a final immunohistochemical score was obtained.

\subsection{Statistical analysis}

Statistical analysis was performed using SPSS 13.0 software package (SPSS Inc., Chicago, IL, USA). Differences be- 
tween groups were evaluated by Chi-square or Fisher's exact tests (two-sided), and Mann-Whitney $U$-test. $P$-values of $<0.05$ were regarded as statistically significant.

\section{Results}

\subsection{COMT expression in pancreatic cancer cell lines}

RT-PCR results showed that COMT was amplified as a fragment of $388 \mathrm{bp}$ in all five pancreatic cancer cell lines (Figure 1A). The COMT protein was also detected in the five pancreatic cancer cell lines by western blotting analysis (Figure 1B). Two bands of 26 and $30 \mathrm{kD}$ conformed respectively to the molecular weights of the two subtypes of COMT proteins, S-COMT and MB-COMT.

\subsection{COMT expression in PDAC and noncancerous tissue}

Five paired samples of PDAC and adjacent normal pancreatic tissue were used for the analysis of COMT expression. RT-PCR revealed that in samples 2 and 3, COMT mRNA expression in PDAC was more than two-fold higher than in the adjacent healthy tissue, while in samples 1,4 and 5, COMT mRNA expression in PDAC was slightly lower than in the adjacent healthy tissues (Figure 2A). In summary, there were no significant differences $(P>0.05)$ between COMT mRNA expression in PDAC and that of paired adjacent healthy tissue, as evaluated by the ratio of the gray value relative to GAPDH.

Next, COMT protein expression in the above five paired samples was analyzed by western blotting. In line with the RT-PCR results, the expression level of COMT in these samples was not significantly different $(P>0.05)$, as evalu-

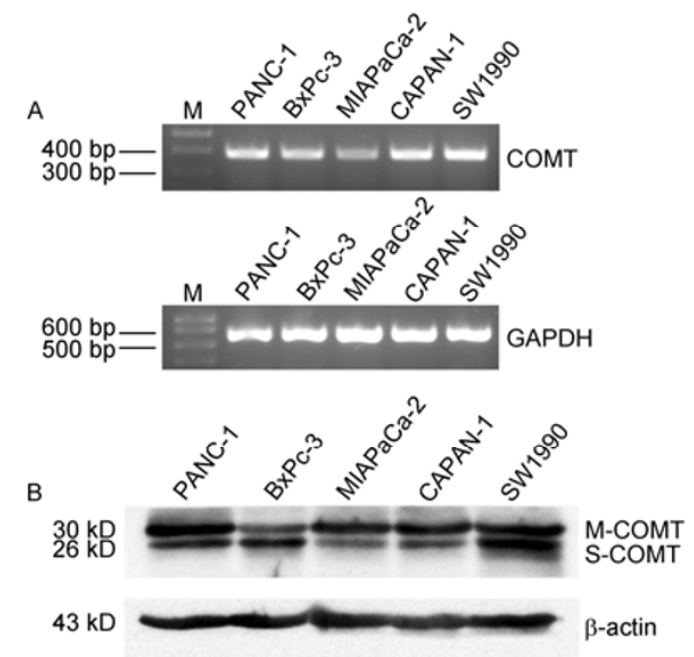

Figure 1 Expression of COMT in AsPC-1, BxPC-3, MIA PaCa-2, Capan-1 and SW1990 pancreatic cancer cell lines. A, Expression of COMT mRNA. GAPDH was used as an internal control. M, marker. B, Expression of COMT protein. $\beta$-actin was used as an internal control.
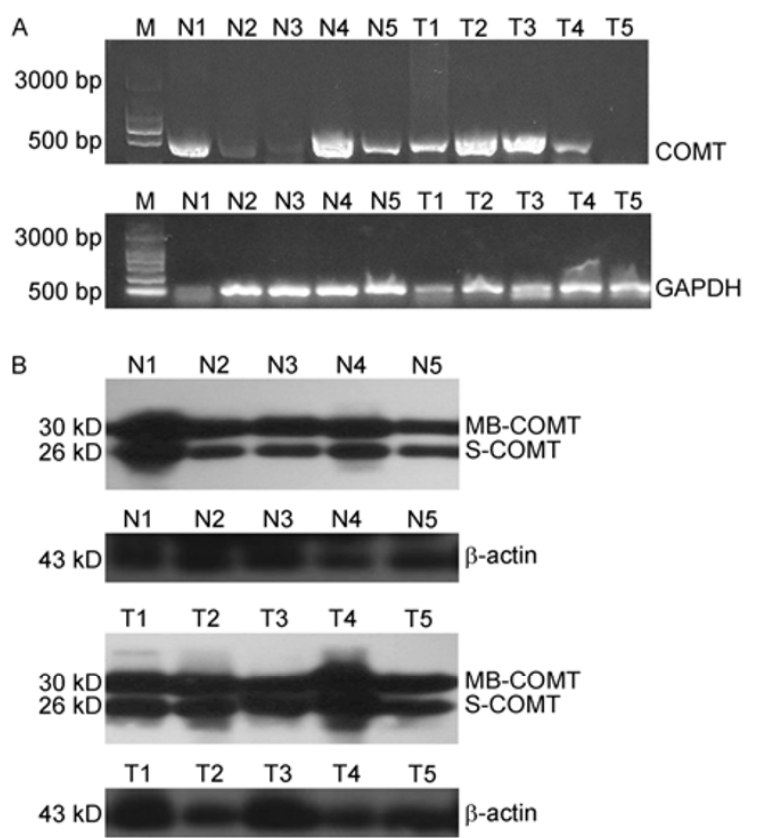

Figure 2 Expression of COMT in five paired clinical samples of PDAC and adjacent nonmalignant pancreatic tissue. A, Expression of COMT mRNA. GAPDH was used as an internal control. M, marker. B, Expression of COMT protein. $\beta$-actin was used as an internal control. N, normal pancreatic tissue; $\mathrm{T}$, tumor.

ated by the ratio of the gray value relative to $\beta$-actin (Figure 2B).

\subsection{Immunohistochemical analysis of COMT expres- sion in PDAC and noncancerous tissue}

We further analyzed COMT protein expression in PDAC and noncancerous tissue by immunohistochemistry. In the 80 paired tissue microarrays, eight paired cases were excluded owing to absent pathology for the PDAC tissue, and the remaining 72 paired cases were included for statistical analysis. In these cases of PDAC, COMT expression was moderate and mostly confined to the cellular cytoplasm (Figure 3A). In nonmalignant pancreatic tissue either adjacent to paired pancreatic cancer ( 72 cases) or paired benign pancreatic tumor (20 cases), we observed negative expression in the ductal epithelium (Figure 3B), moderate cytoplasmic expression of COMT in the pancreatic islet cells, and weak cytoplasmic expression of COMT in the acinar epithelium (Figure 3C).

The staining intensity, percentage of positive cells and score were assessed according to the aforementioned criteria. There were statistically significant differences between pancreatic cancer and nonmalignant pancreatic tissue in staining intensity of COMT $(P=0.003)$, percentage of COMT positive cells $(P<0.001)$ and score $(P<0.001)$ by Chi-square test analysis or Fisher's exact test. Mann-Whitney $U$-test revealed that the percentage of COMT positive cells and the score of PDAC were significantly higher than those of 


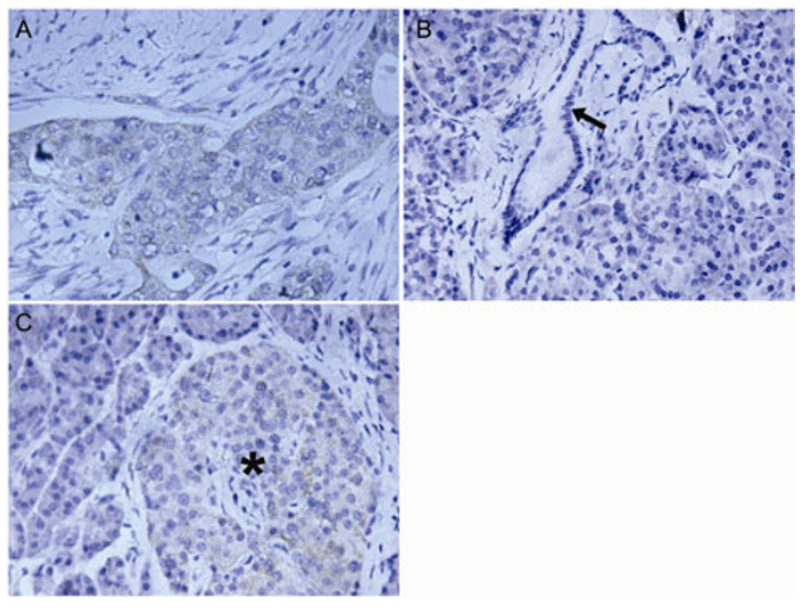

Figure 3 Immunohistochemical analysis of COMT in PDAC and nonmalignant tissue. A, Moderate COMT expression in PDAC (original magnification $\times 400$ ). B, Negative expression of COMT in ductal epithelial cells as indicated by arrow (original magnification $\times 400$ ). C, Weak COMT expression in nonmalignant pancreatic tissue and moderate expression of COMT in islet cells as indicated by asterisk (original magnification $\times 400$ ).

nonmalignant tissue were $(P<0.001$ and $P<0.001$, respectively).

\subsection{Statistical analysis of COMT expression in PDAC with clinicopathologic parameters}

Samples were grouped as COMT ${ }^{\text {High }}$ (immunohistochemical score $\geqslant$ median immunohistochemical score $=4$ ) and as $\mathrm{COMT}^{\text {Low }}$ (immunohistochemical score $<$ median immunohistochemical score=4). Chi-square tests or Fisher's exact tests revealed that clinicopathologic parameters, $\mathrm{T}$ (primary tumor) and TNM stages were significantly correlated with COMT expression status $(P=0.017$ and $P=0.049$, respectively) as shown in Table 1. Using the Mann-Whitney $U$-test, we also found that patients with early $\mathrm{T}$ stages (T1/T2) displayed significantly higher expression of COMT than those with late $\mathrm{T}$ stages $(\mathrm{T} 3 / \mathrm{T} 4)(P=0.022)$. However, there were no significant differences between COMT expression in PDAC cases and other clinicopathologic parameters including the patients' sex, age, $\mathrm{N}$ (regional lymph node), $\mathrm{M}$ (distant metastasis), pathological grade and nerve invasion $(P>0.05)$.

\section{Discussion}

Estrogens are catabolized by hydroxylation reactions. $17 \beta$-Estradiol is metabolized by $16 \alpha$-hydroxylation with the formation of catechol estrogens, 2-hydroxy- and 4-hydroxyestrone/estradiol [16]. COMT catalyzes the process of methylation, which inactivates 16 $\alpha$-, 2-hydroxy-, and 4hydroxy-compounds. 4-Hydroxyestrone and 16 $\alpha$-hydroxyestradiol have estrogenic properties and may be carcinogenic $[17,18]$. In addition, previous studies demonstrated
Table 1 Correlations between COMT expression pattern and clinicopathologic parameters ${ }^{\text {a) }}$

\begin{tabular}{|c|c|c|c|c|}
\hline \multirow{2}{*}{ Characteristics } & \multirow{2}{*}{ Cases $(n)$} & \multicolumn{3}{|c|}{ COMT expression $(\%)$} \\
\hline & & Low & High & $P$-value \\
\hline Sex & & & & 0.382 \\
\hline Male & 47 & $18(38)$ & $29(62)$ & \\
\hline Female & 25 & $7(28)$ & $18(72)$ & \\
\hline Age & & & & 0.458 \\
\hline$\geqslant 58$ median & 36 & $11(31)$ & $25(69)$ & \\
\hline$<58$ median & 36 & $14(39)$ & $22(61)$ & \\
\hline $\mathrm{T}$ & & & & $0.017^{*}$ \\
\hline $\mathrm{T} 1, \mathrm{~T} 2$ & 55 & $15(27)$ & $40(73)$ & \\
\hline $\mathrm{T} 3, \mathrm{~T} 4$ & 17 & $10(59)$ & $7(41)$ & \\
\hline $\mathrm{N}$ & & & & 0.447 \\
\hline No & 38 & $12(32)$ & $26(68)$ & \\
\hline N1 & 27 & $11(41)$ & $16(59)$ & \\
\hline M & & & & 0.296 \\
\hline M0 & 66 & $23(35)$ & $43(65)$ & \\
\hline M1 & 3 & $2(67)$ & $1(33)$ & \\
\hline TNM & & & & $0.049^{*}$ \\
\hline I & 28 & $8(29)$ & $20(71)$ & \\
\hline II & 29 & $11(38)$ & $18(62)$ & \\
\hline III, IV & 6 & $5(83)$ & $1(17)$ & \\
\hline Histological grade & & & & 0.110 \\
\hline MD & 40 & $18(45)$ & $22(55)$ & \\
\hline PD & 24 & $6(25)$ & $18(75)$ & \\
\hline Nerve invasion & & & & 0.083 \\
\hline Yes & 36 & $16(44)$ & $20(56)$ & \\
\hline No & 36 & $9(25)$ & $27(75)$ & \\
\hline
\end{tabular}

a) MD, moderately differentiated; $\mathrm{PD}$, poorly differentiated; *, statistically significant $(P<0.05)$.

that 2-methoxyestrodiol (2-ME), a metabolite of 2-hydroxyestradiol, may be a promising anti-angiogenic and anticancer agent [19]. The anti-proliferative action of 2-ME was specific for cancer cells and was mediated by the induction of programmed cell death or apoptosis [20]. In addition, an epidemiological study in Ontario, Canada, indicated that pancreatic cancer is, at least in part, an estrogen-dependent disease [21]. A population-based case-control study of women from the San Francisco Bay area revealed that age at menopause ( $\geqslant 45$ years vs. $<45$ years) was associated with a 1.8-fold increased risk of pancreatic cancer [22]. These reports suggest that pancreatic cancer might be an estrogen-dependent cancer, and 2-ME may have an inhibitive role in pancreatic cancer.

The results of the present study indicated that COMT was expressed in human pancreatic cancer cell lines at both the mRNA and protein levels, further supporting our previous findings [6,7]. A previous publication demonstrated that in the rat pancreas, bright COMT immunofluorescence was observed in the islet cells, and COMT immunoreactivity was present in insulin-producing $\beta$-cells and somatosta- 
tin-producing D-cells [23]. Our data were in line with these findings as the normal pancreatic tissue COMT expression was more immunoreactive in islet cells than in acinar cells, but there was almost no immunoreactivity in ductal epithelial cells. Therefore, it is possible that the high expression of COMT in islet cells compensates for the expression difference in COMT between PDAC and noncancerous tissue, which would result in the equivalent expression level of COMT mRNA and protein between PDAC and noncancerous tissue.

The present study examined the potential role of COMT in PDAC. Our data revealed a high level of COMT expression in the majority of PDAC compared with noncancerous pancreatic tissue, as determined by immunohistochemistry. Interestingly, an inverse correlation was found between COMT expression and pathological $\mathrm{T}$ stages, in that $\mathrm{COMT}^{\text {Low }}$ expression correlated with late $\mathrm{T}$ stages while $\mathrm{COMT}^{\text {High }}$ expression correlated with early $\mathrm{T}$ stages. These results indicated that $\mathrm{T} 1 / \mathrm{T} 2$ stage tumors, which are limited to the pancreas, had higher levels of COMT than those of T3/T4 stage tumors, which invade the surrounding organs. This suggests that $\mathrm{COMT}^{\text {High }}$ expression may be an early event in the development of PDAC. To date, the association between COMT expression and pancreatic cancer has rarely been reported and no clear relationship has been identified regarding the effect of COMT expression on PDAC. Furthermore, the effect of estrogen on pancreatic cancer remains controversial, based on several clinical trials [24-26]. However, pancreatic cancers showed significantly higher estrogen receptor (ER)- $\beta$ mRNA levels than ER-negative and ER-positive breast cancers, and lower ER- $\alpha$ mRNA expression than ER-negative breast cancers [27]. In more advanced pancreatic cancers, ER- $\beta$ mRNA levels significantly decreased and ER- $\alpha$ mRNA levels increased [27]. In addition, Konduri and Schwarz reported that pancreatic cancer cells expressed functional ERs, generally with a high ER- $\beta$ :ER- $\alpha$ ratio. Estrogen at physiological concentrations predominantly promoted pancreatic cancer cell proliferation in vitro, and cell lines with higher ER- $\beta$ :ER- $\alpha$ ratios tended to show greater responsiveness to ER-modulating agents [28]. Therefore, based on previous reports and the results of the present study, we propose a hypothesis that in the early stages of PDAC, the dominant expression of ER- $\beta$ in pancreatic cancer induces the activation of the estrogen metabolic pathway and the high expression of COMT, which act as promoting factors for PDAC genesis and development. During the development of PDAC, ER- $\beta$ expression decreases and 2-ME increases, the generation of which is directly catalyzed by COMT. Thus, the expression of COMT is reduced either by a less active estrogen metabolic pathway or by a negative feedback mechanism. However, additional studies are required to further elucidate the cancer-promoting mechanism of COMT.

In summary, our study reveals for the first time that
COMT is highly expressed in pancreatic cancer tissue and is significantly associated with early $\mathrm{T}$ stages (T1/T2). Therefore, COMT might serve as a potential biomarker for applied clinical pathology in PDAC.

1 Siegel R, Ward E, Brawley O, et al. Cancer statistics, 2011: the impact of eliminating socioeconomic and racial disparities on premature cancer deaths. CA Cancer J Clin, 2011, 61: 212-236

2 Hidalgo M. Pancreatic cancer. N Engl J Med, 2010, 362: 16051617

3 Gillen S, Schuster T, Meyer Zum Buschenfelde C, et al. Preoperative/neoadjuvant therapy in pancreatic cancer: a systematic review and meta-analysis of response and resection percentages. PLoS Med, 2010, 7: e1000267

4 Kim B J, Lee K T, Moon T G, et al. How do we interpret an elevated carbohydrate antigen 19-9 level in asymptomatic subjects? Dig Liver Dis, 2009, 41: 364-369

5 Duffy M J, Sturgeon C, Lamerz R, et al. Tumor markers in pancreatic cancer: a European Group on Tumor Markers (EGTM) status report. Ann Oncol, 2010, 21: 441-447

6 Pan B, Zhao Y P, Ning L, et al. Proteomic analysis of membrane proteins of endomembrane system in human pancreatic cancer cell (in Chinese). Zhonghua Wai Ke Za Zhi, 2010, 48: 461-465

7 Wang W B, Zhao Y P, Liao Q, et al. Candidate immunogenic membrane antigens of human pancreatic cancer (in Chinese). Zhonghua Wai Ke Za Zhi, 2010, 48: 1412-1415

8 Dempster E L, Mill J, Craig I W, et al. The quantification of COMT mRNA in post mortem cerebellum tissue: diagnosis, genotype, methylation and expression. BMC Med Genet, 2006, 7: 10

9 Wu W M, Zhao Y P, Liao Q, et al. The expression of catechol O-methyltransferase gene in colorectal cancer (in Chinese). Zhonghua Wai Ke Za Zhi, 2010, 48: 535-538

10 Thompson P A, Shields P G, Freudenheim J L, et al. Genetic polymorphisms in catechol-O-methyltransferase, menopausal status, and breast cancer risk. Cancer Res, 1998, 58: 2107-2110

11 Ahsan H, Chen Y, Whittemore A S, et al. A family-based genetic association study of variants in estrogen-metabolism genes COMT and CYP1B1 and breast cancer risk. Breast Cancer Res Treat, 2004, 85: $121-131$

12 Ji Y, Olson J, Zhang J, et al. Breast cancer risk reduction and membrane-bound catechol O-methyltransferase genetic polymorphisms. Cancer Res, 2008, 68: 5997-6005

13 Hirata H, Hinoda Y, Okayama N, et al. COMT polymorphisms affecting protein expression are risk factors for endometrial cancer. Mol Carcinog, 2008, 47: 768-774

14 Freedman N D, Ahn J, Hou L, et al. Polymorphisms in estrogen- and androgen-metabolizing genes and the risk of gastric cancer. Carcinogenesis, 2009, 30: 71-77

15 Kahlert C, Bergmann F, Beck J, et al. Low expression of aldehyde dehydrogenase 1A1 (ALDH1A1) is a prognostic marker for poor survival in pancreatic cancer. BMC Cancer, 2011, 11: 275

16 Huber J C, Schneeberger C, Tempfer C B. Genetic modeling of estrogen metabolism as a risk factor of hormone-dependent disorders. Maturitas, 2002, 41: S55-64

17 Cavalieri E L, Stack D E, Devanesan P D, et al. Molecular origin of cancer: catechol estrogen-3,4-quinones as endogenous tumor initiators. Proc Natl Acad Sci USA, 1997, 94: 10937-10942

18 Yager J D, Liehr J G. Molecular mechanisms of estrogen carcinogenesis. Annu Rev Pharmacol Toxicol, 1996, 36: 203-232

19 Pribluda V S, Gubish E R Jr., Lavallee T M, et al. 2-Methoxyestradiol: an endogenous antiangiogenic and antiproliferative drug candidate. Cancer Metastasis Rev, 2000, 19: 173-179

20 Qanungo S, Basu A, Das M, et al. 2-Methoxyestradiol induces mitochondria dependent apoptotic signaling in pancreatic cancer cells. Oncogene, 2002, 21: 4149-4157

21 Kreiger N, Lacroix J, Sloan M. Hormonal factors and pancreatic 
cancer in women. Ann Epidemiol, 2001, 11: 563-567

22 Duell E J, Holly E A. Reproductive and menstrual risk factors for pancreatic cancer: a population-based study of San Francisco Bay Area women. Am J Epidemiol, 2005, 161: 741-747

23 Karhunen T, Tilgmann C, Ulmanen I, et al. Distribution of catecholO-methyltransferase enzyme in rat tissues. J Histochem Cytochem, 1994, 42: 1079-1090

24 Theve N O, Pousette A, Carlstrom K. Adenocarcinoma of the pancreas-a hormone sensitive tumor? A preliminary report on Nolvadex treatment. Clin Oncol, 1983, 9: 193-197

25 Tonnesen K, Kamp-Jensen M. Antiestrogen therapy in pancreatic carcinoma: a preliminary report. Eur J Surg Oncol, 1986, 12: 69-70

26 Keating J J, Johnson P J, Cochrane A M, et al. A prospective randomised controlled trial of tamoxifen and cyproterone acetate in pancreatic carcinoma. Br J Cancer, 1989, 60: 789-792

27 Iwao K, Miyoshi Y, Ooka M, et al. Quantitative analysis of estrogen receptor-alpha and -beta messenger RNA expression in human pancreatic cancers by real-time polymerase chain reaction. Cancer Lett, 2001, 170: 91-97

28 Konduri S, Schwarz R E. Estrogen receptor beta/alpha ratio predicts response of pancreatic cancer cells to estrogens and phytoestrogens. $\mathbf{J}$ Surg Res, 2007, 140: 55-66

Open Access This article is distributed under the terms of the Creative Commons Attribution License which permits any use, distribution, and reproduction in any medium, provided the original author(s) and source are credited. 